\title{
Terapia medica del dolore neuropatico del soggetto diabetico
}

\author{
Carlo Maria Rotella ${ }^{1}$
}

Accettato: 20 giugno 2020 / Pubblicato online: 16 giugno 2021

(c) The Author(s) 2021

\section{Introduzione}

L'epidemia globale del Diabete Mellito ha portato a un'epidemia delle sue complicanze croniche. Quella che presenta la maggiore prevalenza è la polineuropatia, di cui la più frequente è la polineuropatia simmetrica distale. La Neuropatia Diabetica [1] consiste principalmente in una perdita della funzione sensoriale che inizia nelle estremità inferiori e che è anche caratterizzata da parestesie, dolore e condizioni morbose rilevanti. Nel tempo, almeno il $50 \%$ dei pazienti diabetici sviluppano questa complicanza e un buon controllo glicemico arresta l'evoluzione della neuropatia diabetica nei pazienti con Diabete di tipo 1, ma gli effetti sono meno rilevanti in quelli con Diabete di tipo 2.

\section{Meccanismi del dolore}

Il dolore neuropatico si definisce come un dolore causato da una lesione o condizione morbosa del sistema nervoso somatosensoriale. Circa il 30-50\% dei pazienti con neuropatia diabetica sviluppano dolore, che più frequentemente è un dolore urente spontaneo ai piedi. I pazienti possono anche riferire un'insofferenza allo sfregamento (es. lenzuola) e parestesie. Questi sintomi sensoriali sono spesso accompagnati da perdita della sensibilità e i pazienti riferiscono come un paradosso che i loro piedi sono sempre dolenti benché insensibili al tatto. Il motivo per cui la neuropatia diabetica sviluppi dolore neuropatico non in tutti i pazienti rimane sconosciuto, benché questo rifletta un complesso equilibrio di vulnerabilità che comprende fattori genetici, i circuiti so-

C.M. Rotella

carlomaria.rotella@unifi.it

1 Dipartimento di Scienze Biomediche e Sperimentali "Mario Serio", Unità di Endocrinologia, Università di Firenze, Firenze, Italia matosensoriali, e fattori psicologici in risposta agli agenti stressanti come la disfunzione metabolica del diabete e la gravità della neuropatia.

Gli approcci attuali nel trattamento della neuropatia diabetica si basano sul miglioramento del controllo glicemico (soprattutto nei pazienti con diabete di tipo 1) e sulla modificazione degli stili di vita (soprattutto nei pazienti con diabete di tipo 2) e la terapia del dolore. Tuttavia, una volta instauratasi la complicanza, il raggiungimento dei target di HbA1c ha scarsa influenza nella terapia di questa complicanza e, pertanto, non può essere raccomandata come uno standard terapeutico.

\section{Terapie che mirano a modificare la malattia}

Quattro tipi di farmaci sono stati studiati per curare la patogenesi della neuropatia diabetica che, se pur hanno mostrato una discreta evidenza di efficacia, per molti di essi mancano di evidenze inequivocabili con studi randomizzati e controllati in fase III. Solo l'acido alfa-lipoico, un potente antiossidante, mostra una buona efficacia nel rallentare la comparsa e l'evoluzione della patologia in fase asintomatica o paucisintomatica e per questa molecola esistono le maggiori evidenze.

\section{Terapia del dolore}

Sulla base di molteplici linee guida e articoli di revisione sistematica [2] si evince che i leganti dei canali del calcio a $2 \delta$, gli inibitori della ricaptazione della serotonina e della noradrenalina (SNRIs) e gli antidepressivi triciclici (TCAs) hanno l'evidenza più forte per sostenere il loro uso nel trattamento del dolore nella neuropatia diabetica. Tuttavia, non 
esistono studi di paragone sulla loro efficacia per scegliere il loro uso nella terapia del dolore neuropatico.

Tra gli anticonvulsivi, i leganti a $2 \delta$ Gabapentina e Pregabalina sono ambedue efficaci nel trattamento del dolore. Gabapentina è il più efficace nella maggioranza dei casi, ma non in tutti gli studi. In considerazione del suo profilo farmacocinetico, richiede una titolazione del farmaco. Pregabalina ha un assorbimento dose dipendente all'interno del suo range terapeutico, ma l'efficacia si raggiunge nel 30-50\% dei casi. Per ambedue le molecole esistono effetti collaterali, principalmente confusione e vertigini.

La Duloxetina è un SNRI selettivo che ha dimostrato un'efficacia nel ridurre il dolore in molti trial randomizzati, controllati, con un miglioramento della qualità della vita. Anche la Velafaxina può essere efficace nel trattamento del dolore. L'uso degli SNRI è associato a una gamma di effetti collaterali molto più gravi di quelli osservati con gli anticonvulsivi, quali vertigini, astenia, nausea e insonnia.

Tra gli antidepressivi triciclici, l'Amtriptilina è quello usato più frequentemente, ma la sua efficacia è stata dimostrata in piccoli studi randomizzati, controllati, e anche questi farmaci non sono privi di effetti collaterali. Anche la Nortriptilina e la Desipramina sono stati usati sulla base di evidenze scientifiche meno convincenti.

Benché esista una certa evidenza che l'uso degli oppioidi possa portare a un sollievo del dolore, vi sono rischi di sviluppare dipendenza e altre condizioni di sicurezza nell'uso. Lo stesso si applica per gli analgesici oppiodi atipici. Su questa base, il più recente Position Statement della ADA non raccomanda l'uso di questi farmaci né come prima o seconda linea di intervento.

\section{Conclusione}

La polineuropatia diabetica distale è la complicanza cronica che presenta la maggiore prevalenza e molto frequentemente porta a una sintomatologia dolorosa molto invalidante. Purtroppo, a fronte di queste osservazioni, la terapia del dolore non presenta al momento una possibilità di intervento terapeutico che garantisca una risoluzione certa della sintomatologia.

Funding Note Open access funding provided by Università degli Studi di Firenze within the CRUI-CARE Agreement.

Conflitto di interesse L'autore Carlo Maria Rotella dichiara di non avere conflitti di interesse.

Consenso informato Lo studio presentato in questo articolo non ha richiesto sperimentazione umana.

Studi sugli animali L'autore di questo articolo non ha eseguito studi sugli animali.

Nota della casa editrice Springer Nature rimane neutrale in riguardo alle rivendicazioni giurisdizionali nelle mappe pubblicate e nelle affiliazioni istituzionali.

Open Access This article is licensed under a Creative Commons Attribution 4.0 International License, which permits use, sharing, adaptation, distribution and reproduction in any medium or format, as long as you give appropriate credit to the original author(s) and the source, provide a link to the Creative Commons licence, and indicate if changes were made. The images or other third party material in this article are included in the article's Creative Commons licence, unless indicated otherwise in a credit line to the material. If material is not included in the article's Creative Commons licence and your intended use is not permitted by statutory regulation or exceeds the permitted use, you will need to obtain permission directly from the copyright holder. To view a copy of this licence, visit http://creativecommons.org/licenses/by/4.0/.

\section{Bibliografia}

1. Feldman EL, Callaghan BC, Pop-Busui R et al (2019) Diabetic neuropathy. Nat Rev Dis Primers 5:41-59

2. Cakici N, Fakkel TM, van Neck JW et al (2016) Systematic review of treatments for diabetic peripheral neuropathy. Diabet Med 33:1466-1476 